\title{
Limits of Natural Logic
}

\author{
Lauri Karttunen \\ Center for the Study of Language and Information \\ 210 Panama St. Stanford, CA 94305, USA \\ Stanford University \\ laurikestanford.edu
}

\section{Abstract of the Invited Talk}

Natural Logic attempts to do formal reasoning in natural language in a proof-theoretic way making use of the syntactic structure and the semantic properties of lexical items and constructions. It goes beyond trying to establish who did what to whom to yield further inferences. Natural Logic contrasts with approaches that involve a translation from a natural into a formal language such as predicate calculus or a higher-order logic that is sometimes deemed a more appropriate basis for reasoning although difficult to implement efficiently. Both approaches have been used in current work on computational semantics.

Natural Logic goes back to the Aristotelian syllogisms; computing meaning representations in an artificial language emerged in more recent times with the advances in logic and model-theoretic approaches to semantics.

This talk will start with a brief history of Natural Logic from its origins to the most recent work on monotonicity, MacCartney's lexical relations and implicatives. This part is a success story. We will then illustrate four areas that seem to fall outside the classical Natural Logic framework: presuppositions, soft inferences, the consonance/dissonance effect of evaluative adjectives, and the double meaning of the be lucky to VP in the future tense. These cases are also affected by pragmatic considerations: opinions about the world, assignments of beliefs to people, and even by the perceived communicative intent of the speaker.
Natural Logic needs to be augmented with pragmatics to make it a richer theory of Natural Reasoning.

\section{References}

MacCartney, Bill and Christopher Manning (2009). An extended model of natural logic. In Proceedings of the Eighth International Conference on Computational Semantics (IWCS-8), Tilburg, Netherlands, January 2009, pages 140-156.

Icard III, Thomas (2012). Inclusion and Exclusion in Natural Language. Studia Logica 100 (4):705725.

Icard III, Thomas and Larry Moss (2014). Recent Progress on Monotonicity. Linguistic Issues in Language Technology 9.

Karttunen, Lauri (2012). Simple and Phrasal Implicatives. Proceedings of *SEM 2012: The First Joint Conference on Lexical and Computational Semantics. Volume I, pages 124-131.

Karttunen, Lauri. 2013. You will be lucky to break even. In From quirky case to representing space. Papers in honor of Annie Zaenen, pages 167180. Stanford, CA: CSLI Publications.

Karttunen, Lauri, Stanley Peters, Annie Zaenen, and Cleo Condoravdi (2014). The Chameleon-like Nature of Evaluative Adjectives. Empirical Issues in Syntax and Semantics 10, pages 233-250.

van Benthem, Johan (2008). A Brief History of Natural Logic. ILLC Publications, All Series, 2008, PP-2008-05. 